\title{
Constraints on the gas content of the Fomalhaut debris belt
}

\section{Can gas-dust interactions explain the belt's morphology ${ }^{\star}, \star \star$}

\author{
G. Cataldi ${ }^{1,2}$, A. Brandeker ${ }^{1,2}$, G. Olofsson ${ }^{1,2}$, C. H. Chen ${ }^{3}$, W. R. F. Dent ${ }^{4}$, I. Kamp ${ }^{5}$, \\ A. Roberge ${ }^{6}$, and B. Vandenbussche ${ }^{7}$
}

\author{
1 AlbaNova University Centre, Stockholm University, Department of Astronomy, 10691 Stockholm, Sweden \\ e-mail: gianni.cataldi@astro.su.se \\ 2 Stockholm University Astrobiology Centre, 10691 Stockholm, Sweden \\ 3 Space Telescope Science Institute, 3700 San Martin Drive, Baltimore, MD 21218, USA \\ 4 ALMA SCO, Alonso de Cordova 3107, Vitacura, Casilla 763 0355, Santiago, Chile \\ 5 Kapteyn Astronomical Institute, University of Groningen, Postbus 800, 9700 AV Groningen, The Netherlands \\ 6 Goddard Center for Astrobiology, Goddard Space Flight Center, Greenbelt, MD 20771, USA \\ 7 Institute of Astronomy KU Leuven, Celestijnenlaan 200D, 3001 Leuven, Belgium
}

Received 12 November 2014 / Accepted 18 December 2014

\section{ABSTRACT}

\begin{abstract}
Context. The 440 Myr old main-sequence A-star Fomalhaut is surrounded by an eccentric debris belt with sharp edges. This sort of a morphology is usually attributed to planetary perturbations, but the orbit of the only planetary candidate detected so far, Fomalhaut $b$, is too eccentric to efficiently shape the belt. Alternative models that could account for the morphology without invoking a planet are stellar encounters and gas-dust interactions.

Aims. We aim to test the possibility of gas-dust interactions as the origin of the observed morphology by putting upper limits on the total gas content of the Fomalhaut belt.

Methods. We derive upper limits on the C II $158 \mu \mathrm{m}$ and O I $63 \mu \mathrm{m}$ emission by using non-detections from the Photodetector Array Camera and Spectrometer (PACS) onboard the Herschel Space Observatory. Line fluxes are converted into total gas mass using the non-local thermodynamic equilibrium (non-LTE) code RADEX. We consider two different cases for the elemental abundances of the gas: solar abundances and abundances similar to those observed for the gas in the $\beta$ Pictoris debris disc.

Results. The gas mass is shown to be below the millimetre dust mass by a factor of at least $\sim 3$ (for solar abundances) respectively $\sim 300$ (for $\beta$ Pic-like abundances).

Conclusions. The lack of gas co-spatial with the dust implies that gas-dust interactions cannot efficiently shape the Fomalhaut debris belt. The morphology is therefore more likely due to a yet unseen planet (Fomalhaut c) or stellar encounters.
\end{abstract}

Key words. circumstellar matter - planetary systems - stars: individual: Fomalhaut - methods: observational - hydrodynamics infrared: general

\section{Introduction}

Fomalhaut is one of the best studied examples out of several hundred main-sequence stars known to be surrounded by dusty discs commonly known as debris discs. The presence of circumstellar material around this nearby (7.7 pc; van Leeuwen 2007), $440 \pm 40$ Myr old (Mamajek 2012) A3V star was first inferred by the detection of an infrared excess above the stellar photosphere due to thermal emission from micron-sized dust grains (Aumann 1985). The dusty debris belt around Fomalhaut has been resolved at infrared wavelengths with Spitzer (Stapelfeldt et al. 2004) and the Herschel Photodetector Array Camera and Spectrometer (PACS; Acke et al. 2012). The dust is thought to be derived from continuous collisions of larger planetesimals or cometary objects (Backman \& Paresce 1993). To trace these parent bodies, observations at longer wavelengths

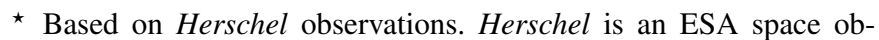
servatory with science instruments provided by European-led Principal Investigator consortia and with important participation from NASA.

$\star \star$ Table 2 is available in electronic form at http://www . aanda.org
}

sensitive to millimetre-sized grains are used. Millimetre-sized grains are less affected by radiation pressure and thus more closely follow the distribution of the parent bodies. Holland et al. (1998) were the first to resolve the Fomalhaut belt in the sub-millimetre. Recent data from the Atacama Large Millimeter/submillimeter Array (ALMA) show a remarkably narrow belt with a semi-major axis $a \sim 140$ AU (Boley et al. 2012, hereafter B12).

The belt has also been observed in scattered star light with the Hubble Space Telescope (Kalas et al. 2005, 2013). Two key morphological characteristics emerging from the Kalas et al. (2005) observations are i) the ring's eccentricity of $e \sim 0.1$; and ii) its sharp inner edge. Both features have been seen as evidence for a planet orbiting the star just inside the belt (e.g. Quillen 2006). A candidate planetary body (Fomalhaut b) was detected (Kalas et al. 2008), but subsequent observations showed that its orbit is highly eccentric ( $e=0.8 \pm 0.1)$, making it unlikely to be responsible for the observed morphology (Kalas et al. 2013; Beust et al. 2014; Tamayo 2014). There remains the possibility that a different, hereto unseen planet is shaping 
the belt; infrared surveys were only able to exclude planets with masses larger than a Jupiter mass (Kalas et al. 2008; Marengo et al. 2009; Janson et al. 2012, 2015). Actually, the extreme orbit of Fomalhaut $b$ might be a natural consequence of the presence of an additional planet in a moderately eccentric orbit (Faramaz et al. 2015). Alternatively, the observed belt eccentricity may be caused by stellar encounters (e.g. Larwood \& Kalas 2001; Jalali $\&$ Tremaine 2012). In particular, Fomalhaut is part of a wide triple system. Shannon et al. (2014) recently showed that secular interactions or close encounters with one of the companions could result in the observed belt eccentricity.

We focus on yet another candidate mechanism to explain the observed morphology. It is known that gas-dust interactions can result in a clumping instability, organising the dust into narrow rings (Klahr \& Lin 2005; Besla \& Wu 2007). Lyra \& Kuchner (2013) recently presented the first 2D simulations of this instability and found that some rings develop small eccentricities, resulting in a morphology similar to that observed for the Fomalhaut debris belt. The mechanism obviously requires the presence of gas beside the dust (typically, a dust-to-gas ratio of $\epsilon \lesssim 1$ is required), but we know from objects such as $\beta$ Pictoris (e.g. Olofsson et al. 2001) or 49 Ceti (e.g. Hughes et al. 2008) that debris discs are not always devoid of gas. We test the applicability of the clumping instability to the case of Fomalhaut by searching for C II $158 \mu \mathrm{m}$ and O I $63 \mu \mathrm{m}$ gas emission using Herschel PACS. We do not detect any of the emission lines and use our data to put stringent upper limits on the gas content of the Fomalhaut debris belt.

\section{Observations and data reduction}

Fomalhaut was observed using PACS (Poglitsch et al. 2010) onboard the Herschel Space Observatory (Pilbratt et al. 2010). The integral field unit PACS consists of 25 spatial pixels (spaxels), each covering $9.4^{\prime \prime} \times 9.4^{\prime \prime}$ on the sky. We used PACS in line spectroscopy mode (PacsLineSpec) to observe the C II $158 \mu \mathrm{m}$ and the O I $63 \mu \mathrm{m}$ line regions (observation IDs 1342257220 and 1342210402 respectively). We observed in chop/nod mode. Figure 1 shows the placement of the PACS spaxels relative to the Fomalhaut debris belt in the case of the C II observations. We reduced the data using the "background normalisation" pipeline script within the Herschel interactive processing environment (HIPE) version 12.0 (Ott 2010). The background normalisation pipeline is recommended for faint sources or long observations since it corrects more efficiently for detector drifts compared to the "calibration block" pipeline. The pipeline performs bad pixel flagging, cosmic ray detection, chop on/off subtraction, spectral flat fielding, and re-binning. We choose a rebinning with oversample $=4$ (thus gaining spectral resolution ${ }^{1}$ by making use of the redundancy in the data) and upsample $=1$ (thus keeping the individual data points independent). Finally, the pipeline averages the two nodding positions and calibrates the flux. The noise in the PACS spectra is lowest at the centre of the spectral range and increases versus the spectral edges. We discard the very noisy spectral edges. We then subtract the continuum by fitting linear polynomials to the spectra, where the line region is masked. The pipeline generated noise estimate

\footnotetext{
1 With this choice of re-binning, the resolution is $\sim 60 \mathrm{~km} \mathrm{~s}^{-1}$ for the C II data and $\sim 22 \mathrm{~km} \mathrm{~s}^{-1}$ for the O I data.
}

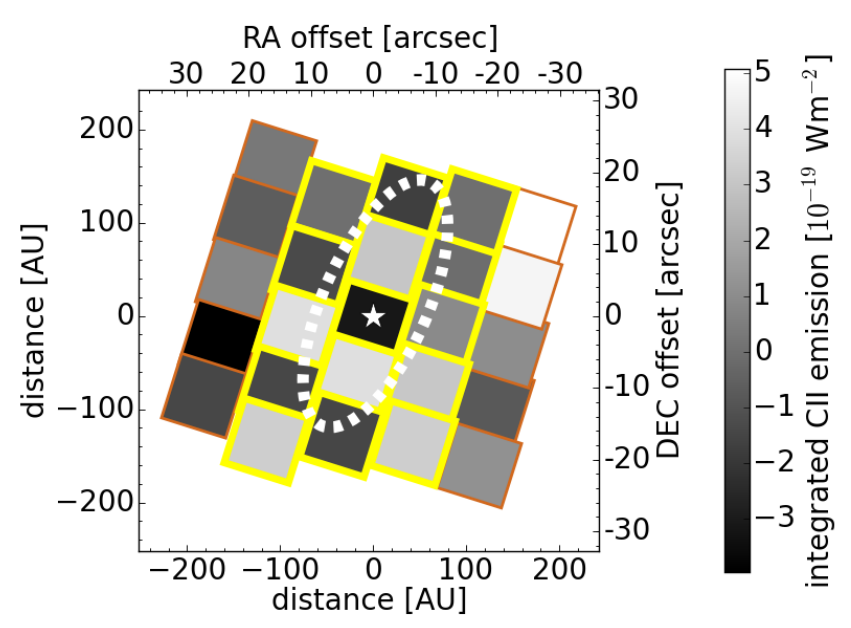

Fig. 1. Placement of the 25 PACS spaxels relative to Fomalhaut (marked by the white star) in the case of the C II observations. The grey scale indicates the integrated C II emission for each spaxel within a window of width equal to $2.5^{*} F W H M$ of the line. The ellipse shows the location of the peak density of $\mathrm{mm}$ grains according to the best-fit model by $\mathrm{B} 12$. The corresponding figure for the $\mathrm{O}$ I data looks very similar. The 15 spaxels with a yellow bold edge were summed to produce Fig. 2.

"stddev" is used to give the highest weight to the data points at the centre of the spectral range ${ }^{2}$.

\section{Results and analysis}

\subsection{Upper limits on the $\mathrm{C} I \mathrm{I}$ and $\mathrm{OI}$ emission from PACS}

Whereas both lines remain undetected, we do detect the dust continuum at both wavelengths in the spaxels covering the belt. At $63 \mu \mathrm{m}$, we see additional continuum emission at the position of the star. Within the absolute flux calibration accuracy of PACS, the total detected continuum is consistent with the photometry presented by Acke et al. (2012).

We adopt a forward modelling strategy to compute upper limits on the line flux. We simulate PACS observations from a map of the line intensity on the sky. The PACS Spectrometer beams (version 3$)^{3}$ are used to calculate the integrated line flux registered in each spaxel from the input map. The line is assumed to be unresolved. Thus the computed flux is assumed to be contained in a Gaussian-shaped line with a full width at half maximum (FWHM) determined by the PACS instrument ${ }^{4}$. These simulated spectra can then be compared to the real observations. The input sky map is constructed under the assumption that the gas is co-spatial with the dust, since we are interested in constraining the possibility of gas-dust interactions. We take the best-fit model of the distribution of mm grains derived by B12 as a description of the line luminosity density (energy from line emission per volume) and derive a sky map assuming optically thin emission. The only free parameter in the model is a global, positive scaling of the sky map, determining the total flux received from the belt. We adopt a simple Bayesian approach with

\footnotetext{
2 Even if the noise estimate delivered by the pipeline probably underestimates the true uncertainty, it is still useful for fitting the continuum, since it describes the relative change of the noise over the spectral range. 3 http://herschel.esac.esa.int/twiki/bin/view/Public/ PacsCalibrationWeb\#PACS_spectrometer_calibration

$40.126 \mu \mathrm{m}$ for the $\mathrm{C}$ II $158 \mu \mathrm{m}$ line and $0.018 \mu \mathrm{m}$ for the O I $63 \mu \mathrm{m}$ line; see Sect. 4.7.1 of the PACS Observer's Manual, http://herschel. esac. esa. int/Docs/PACS/pdf/pacs_om.pdf
} 
Table 1. Upper limits (99\% confidence level) on line fluxes.

\begin{tabular}{cc}
\hline \hline Line & $\begin{array}{c}\text { Flux } \\
\left(\mathrm{W} \mathrm{m}^{-2}\right)\end{array}$ \\
\hline C II $158 \mu \mathrm{m}$ & $<2.2 \times 10^{-18}$ \\
O I $63 \mu \mathrm{m}$ & $<1.0 \times 10^{-17}$ \\
\hline
\end{tabular}

a flat prior for the scaling factor. Assuming Gaussian noise for the PACS data points, a posterior probability distribution can be derived, which is used to compute an upper limit on the scaling factor and thus on the total line emission. The noise is estimated for each spaxel individually from two $2.5 * F W H M$ wide spectral windows, placed sufficiently far from the line centre so to avoid any potential line emission. However, the windows are also sufficiently far from the noisy edges of the spectral range. Thus we do not significantly overestimate the noise in the line region itself.

Table 1 shows upper limits on the total line emission of gas co-spatial with the dust at $99 \%$ confidence level. The C II line gives a significantly stronger upper limit because of the lower noise level in these data. We also estimated upper limits using Monte Carlo simulations by repeatedly fitting our model to new realisations of the data with added noise. This approach gives upper limits that are smaller by $\sim 5 \%$ for C II and $\sim 20 \%$ for O I compared to the Bayesian calculation. We state the more conservative Bayesian values here. Compared to co-adding flux from the spaxels covering the belt (Fig. 1), our approach using a model of the spatial distribution allows for a stronger upper limit (Fig. 2). This is expected since more information is used to constrain the maximum flux compatible with the data.

\subsection{Upper limits on the total gas mass in the Fomalhaut debris belt}

We use the non-local thermodynamic equilibrium (non-LTE) excitation and radiative transfer code RADEX (van der Tak et al. 2007) to convert the upper limits on the C II and O I line emission (Table 1) into gas masses. The emission lines are assumed to be excited by collisions with atomic hydrogen and electrons. We do not attempt to calculate the thermal balance, but instead consider a range of kinetic gas temperatures between $48 \mathrm{~K}$ (the dust temperature, B12) and $10^{4} \mathrm{~K}$, which is the temperature where collisional ionisation of both $\mathrm{C}$ and $\mathrm{O}$ becomes important. The $\mathrm{C} / \mathrm{O}$ ratio is fixed to solar. For the abundance of the other elements, we consider two cases: solar abundances (Lodders 2003) and abundances as observed in the $\beta$ Pictoris gaseous debris disc, where the gas mass is dominated by $\mathrm{C}$ and $\mathrm{O}$ (Cataldi et al. 2014; Brandeker et al. 2015). The latter choice of abundances is further motivated by a recent study of the 49 Ceti debris disc, suggesting a C-rich gas similar to $\beta$ Pic (Roberge et al. 2014).

For a given temperature, we search the amount of gas that is reproducing either of the upper limits (C II or O I). Usually, the gas mass is limited by the $\mathrm{C}$ II flux. The $\mathrm{O}$ I flux is the limiting factor only for kinetic temperatures in excess of $150 \mathrm{~K}$ and solar abundances (see Fig. 3). The densities of the collision partners $\left(\mathrm{e}^{-}\right.$and $\left.\mathrm{H}\right)$ determine the excitation of the emission lines and therefore the total amount of gas necessary to reproduce a certain flux, but the total amount of gas also implies certain values for the $\mathrm{H}$ and $\mathrm{e}^{-}$densities. Therefore, to construct a self-consistent model, an iterative approach is necessary. Starting with a guess of the $\mathrm{e}^{-}$and $\mathrm{H}$ densities, we use RADEX to compute the amount of $\mathrm{C}^{+}$(or $\mathrm{O}$ if $\mathrm{O} \mathrm{I}$ is limiting the total gas mass) reproducing the corresponding flux upper limit. Next, the ionisation fraction of

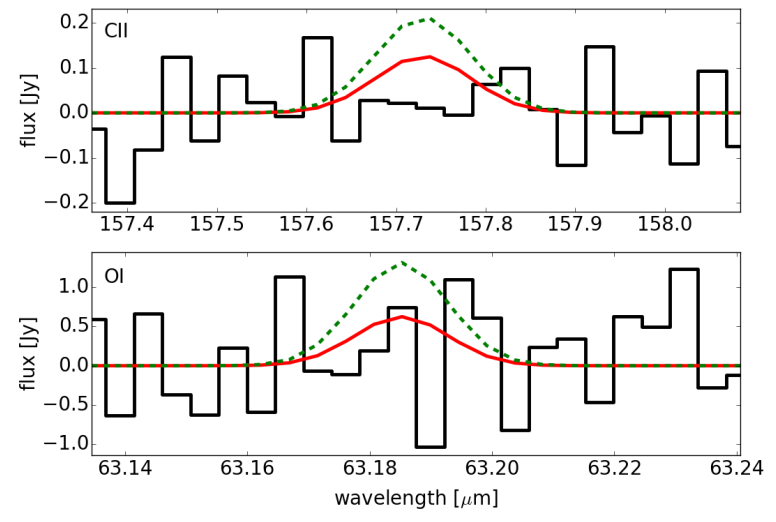

Fig. 2. Line fluxes (C II and OI) obtained when co-adding spaxels marked with bold edges in Fig. 1. A 99\% confidence level upper limit was derived by integrating over a $2 * F W H M$ wide wavelength range centred at the line wavelength and propagating the error on the integrated flux. The dashed line shows the Gaussian profile containing this upper limit flux. The plain line shows the profile obtained by co-adding the same spaxels containing our simulated spectra corresponding to the Bayesian upper limit, demonstrating the stronger limit obtained with our detailed model.

$\mathrm{C}$ is estimated. We assume equilibrium between photoionisation and recombination. Ionising photons from two different sources are included: Fomalhaut's photosphere from an ATLAS9 model (Castelli \& Kurucz 2004) with $T_{\text {eff }}=8500 \mathrm{~K}, \log g=4.0$ and $\log Z=0$, and the interstellar UV field (taken from Draine 1978), where the latter is the dominant component. Ionisation by cosmic rays is negligible. A simplifying assumption is that all electrons are coming from the ionisation of C, i.e. $n_{\mathrm{C}}=n_{\mathrm{e}^{-}}$. This should be an excellent approximation if the gas has $\beta$ Piclike abundances. For solar abundances, we are likely underestimating the electron density because of the presence of highly ionised elements such as $\mathrm{Mg}$ or Fe. This only affects the ionisation calculation, but not the excitation of the lines, since for solar abundances, $\mathrm{H}$ is dominating the collisional excitation ${ }^{5}$. The ionisation fraction of $\mathrm{C}$ is giving us the total amount of $\mathrm{C}$ and $\mathrm{e}^{-}$in the model. The total gas mass (and in particular the $\mathrm{H}$ density) can then be derived from the assumed elemental abundances, where elements up to atomic number $Z=38$ are considered (hydrogen through strontium). We iterate until the $\mathrm{H}$ and $\mathrm{e}^{-}$input densities are equal to the output densities implied by the total gas mass. We assume $\mathrm{O}$ to be completely neutral in our models because of its high ionisation potential. This is justified by computing the ionisation of $\mathrm{O}$ for various electron densities and temperatures, showing that $\mathrm{O}$ remains largely neutral for $n_{\mathrm{e}} \gtrsim 10^{-2} \mathrm{~cm}^{-3}$.

While RADEX assumes a homogeneous medium, we calculated the upper limits on the line emission using the B12 dust profile as a description of the line luminosity density. We convert a gas mass output from RADEX to a density by distributing the mass according to the B12 profile and taking the peak density. For example, the $\mathrm{C}^{+}$mass from RADEX implies an $\mathrm{e}^{-}$density via the peak density of the B12 density profile. Changing this massto-density conversion by e.g. taking only half the peak density has only a minor effect on our results.

Table 2 shows the upper limits on the gas mass and corresponding lower limits on the dust-to-gas ratio derived for the two elemental abundances considered. We also list upper limits

5 In the case of the H-poor $\beta$ Pic-like abundances, collisional excitation is completely dominated by $\mathrm{e}^{-}$. 


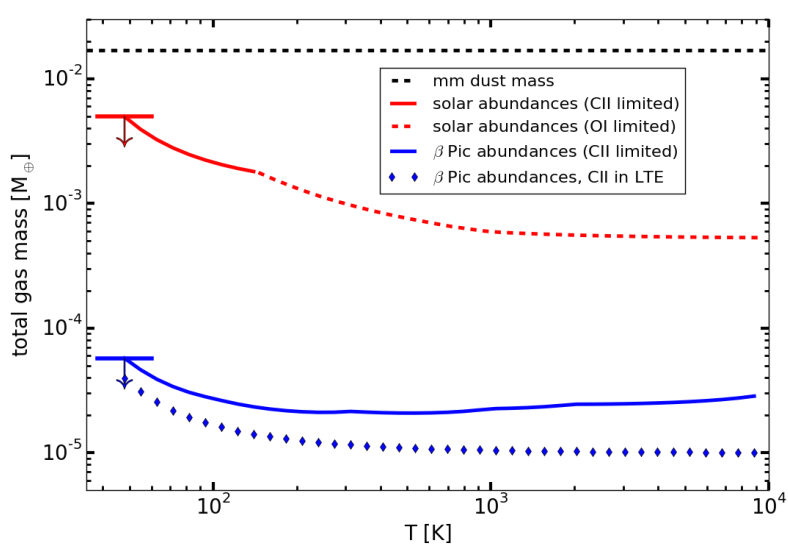

Fig. 3. Total gas mass as a function of the kinetic temperature, derived from models reproducing either the $\mathrm{C}$ II or O I flux limit from Table 1 (as indicated in the legend). The $\mathrm{C} / \mathrm{O}$ ratio is fixed to solar. The gas masses are derived using the non-LTE code RADEX for both solar and $\beta$ Pic-like elemental abundances. In addition, the gas mass derived from a simple LTE calculation is shown for $\beta$ Pic-like abundances. The total gas mass remains below the mm dust mass (horizontal dashed line) over the entire temperature range. We take the gas masses at the lowest kinetic temperature as our upper limits (indicated by an arrow and shown in Table 2).

on the $\mathrm{C}$ and $\mathrm{O}$ mass, the range of collision partner densities occurring and the maximum column densities, showing that the emission is optically thin. Figure 3 shows the gas mass as a function of the kinetic temperature. The upper limit on the gas mass comes from the lowest kinetic temperature $(T=48 \mathrm{~K})$ and is below the millimetre dust mass derived by B12. For illustration, we also performed a simple calculation of the amount of $\mathrm{C}$ necessary to reproduce the upper limit on the C II flux in the case of LTE for $\beta$ Pic-like abundances. It is seen that LTE is not a very accurate approximation, demonstrating the need for a non-LTE code like RADEX for the type of environment investigated here.

\section{Summary and conclusion}

Gas-dust interactions have been shown to concentrate dust into narrow, eccentric rings via a clumping instability. A necessary condition for the instability to develop is a dust-to-gas ratio $\epsilon \lesssim 1$ (Lyra \& Kuchner 2013), although the instability is expected to be maximised for $\epsilon \approx 0.2$ (W. Lyra 2014, priv. comm.). We know from e.g. $\beta$ Pictoris or 49 Ceti that some debris discs contain gas such that $\epsilon$ is indeed of the order of unity. Prior to the present work, the only information on the gas content of the Fomalhaut debris belt came from non-detections of CO. Dent et al. (1995) used a non-detection of the $J=3-2$ transition to put upper limits on the CO gas mass assuming LTE. Recently, Matrà et al. (2014) used an ALMA non-detection of the same transition to place a $3 \sigma$ upper limit of $\sim 5 \times 10^{-4} M_{\oplus}$ on the $\mathrm{CO}$ content of the Fomalhaut belt (i.e. a dust-to- $\mathrm{CO}$ ratio z30), taking the crucial importance of non-LTE effects into account. However, since $\mathrm{CO}$ is expected to be photo-dissociated by stellar or interstellar UV photons on a short timescale compared to the lifetime of the system (Kamp \& Bertoldi 2000; Visser et al. 2009), the absence of CO does not strongly constrain the total gas mass. Constraints on the atomic gas mass are more useful. We derived upper limits on the atomic gas mass using non-detections of $\mathrm{C}$ II and $\mathrm{OI}$ emission and assuming either solar abundances or abundances as those observed in the $\beta$ Pic debris disc. The total gas mass is shown to be smaller than the dust mass. Because of the low gas content, gas-dust interactions are not expected to be working efficiently in the Fomalhaut belt. Therefore, the data indirectly suggest a second, yet unseen planet Fomalhaut $\mathrm{c}$ or stellar encounters as the cause for the morphology of the Fomalhaut debris belt.

\begin{abstract}
Acknowledgements. We would like to thank the referee, Jane Greaves, for useful and constructive comments that helped to clarify this manuscript. We also thank Elena Puga from the Herschel Science Centre Helpdesk for support with the Herschel beam products. This research has made use of the SIMBAD database (operated at CDS, Strasbourg, France), the NIST Atomic Spectra Database, the NORAD-Atomic-Data database and NASA's Astrophysics Data System. PACS has been developed by a consortium of institutes led by MPE (Germany) and including UVIE (Austria); KU Leuven, CSL, IMEC (Belgium); CEA, LAM (France); MPIA (Germany); INAF-IFSI/OAA/OAP/OAT, LENS, SISSA (Italy); IAC (Spain). This development has been supported by the funding agencies BMVIT (Austria), ESA-PRODEX (Belgium), CEA/CNES (France), DLR (Germany), ASI/INAF (Italy), and CICYT/MCYT (Spain).
\end{abstract}

\section{References}

Acke, B., Min, M., Dominik, C., et al. 2012, A\&A, 540, A125 Aumann, H. H. 1985, PASP, 97, 885

Backman, D. E., \& Paresce, F. 1993, in Protostars and Planets III, eds. E. H. Levy, \& J. I. Lunine, 1253

Besla, G., \& Wu, Y. 2007, ApJ, 655, 528

Beust, H., Augereau, J.-C., Bonsor, A., et al. 2014, A\&A, 561, A43

Boley, A. C., Payne, M. J., Corder, S., et al. 2012, ApJ, 750, L21

Brandeker, A., Olofsson, G., Vandenbussche, B., et al. 2015, A\&A, submitted

Castelli, F., \& Kurucz, R. L. 2004 [arXiv: astro-ph/0405087]

Cataldi, G., Brandeker, A., Olofsson, G., et al. 2014, A\&A, 563, A66

Dent, W. R. F., Greaves, J. S., Mannings, V., Coulson, I. M., \& Walther, D. M. 1995, MNRAS, 277, L25

Draine, B. T. 1978, ApJS, 36, 595

Faramaz, V., Beust, H., Augereau, J.-C., Kalas, P., \& Graham, J. R. 2015, A\&A, 573, A87

Holland, W. S., Greaves, J. S., Zuckerman, B., et al. 1998, Nature, 392, 788

Hughes, A. M., Wilner, D. J., Kamp, I., \& Hogerheijde, M. R. 2008, ApJ, 681, 626

Jalali, M. A., \& Tremaine, S. 2012, MNRAS, 421, 2368

Janson, M., Carson, J. C., Lafrenière, D., et al. 2012, ApJ, 747, 116

Janson, M., Quanz, S. P., Carson, J. C., et al. 2015, A\&A, in press, DOI: $10.1051 / 0004-6361 / 201424944$

Kalas, P., Graham, J. R., \& Clampin, M. 2005, Nature, 435, 1067

Kalas, P., Graham, J. R., Chiang, E., et al. 2008, Science, 322, 1345

Kalas, P., Graham, J. R., Fitzgerald, M. P., \& Clampin, M. 2013, ApJ, 775, 56

Kamp, I., \& Bertoldi, F. 2000, A\&A, 353, 276

Klahr, H., \& Lin, D. N. C. 2005, ApJ, 632, 1113

Larwood, J. D., \& Kalas, P. G. 2001, MNRAS, 323, 402

Lodders, K. 2003, ApJ, 591, 1220

Lyra, W., \& Kuchner, M. 2013, Nature, 499, 184

Mamajek, E. E. 2012, ApJ, 754, L20

Marengo, M., Stapelfeldt, K., Werner, M. W., et al. 2009, ApJ, 700, 1647

Matrà, L., Panić, O., Wyatt, M. C., \& Dent, W. R. F. 2014, MNRAS, in press [arXiv: 1412.2757]

Olofsson, G., Liseau, R., \& Brandeker, A. 2001, ApJ, 563, L77

Ott, S. 2010, in Astronomical Data Analysis Software and Systems XIX, eds. Y. Mizumoto, K.-I. Morita, \& M. Ohishi, ASP Conf. Ser., 434, 139

Pilbratt, G. L., Riedinger, J. R., Passvogel, T., et al. 2010, A\&A, 518, L1

Poglitsch, A., Waelkens, C., Geis, N., et al. 2010, A\&A, 518, L2

Quillen, A. C. 2006, MNRAS, 372, L14

Roberge, A., Welsh, B. Y., Kamp, I., Weinberger, A. J., \& Grady, C. A. 2014, ApJ, 796, L11

Shannon, A., Clarke, C., \& Wyatt, M. 2014, MNRAS, 442, 142

Stapelfeldt, K. R., Holmes, E. K., Chen, C., et al. 2004, ApJS, 154, 458

Tamayo, D. 2014, MNRAS, 438, 3577

van der Tak, F. F. S., Black, J. H., Schöier, F. L., Jansen, D. J., \& van Dishoeck, E. F. 2007, A\&A, 468, 627

van Leeuwen, F. 2007, Hipparcos, the New Reduction of the Raw Data, Astrophys. Space Sci. Libr., 350

Visser, R., van Dishoeck, E. F., \& Black, J. H. 2009, A\&A, 503, 323

Page 5 is available in the electronic edition of the journal at http://www . aanda.org 
G. Cataldi et al.: Constraints on the gas content of the Fomalhaut debris belt

Table 2. Upper limits on the total gas mass (and corresponding lower limits on the gas-to-dust ratio), the $\mathrm{C}$ mass and the $\mathrm{O}$ mass.

\begin{tabular}{ccccccccccc}
\hline \hline Abundances & $M_{\text {gas }}$ & $\epsilon^{a}$ & $M_{\mathrm{C}}$ & $M_{\mathrm{O}}$ & \multicolumn{2}{c}{$\mathrm{e}^{-}$density } & \multicolumn{2}{c}{$\mathrm{H}$ density } & $N_{\max }{ }^{d}\left(\mathrm{C}^{+}\right)$ & $N_{\max }{ }^{d}(\mathrm{O})$ \\
& $\left(M_{\oplus}\right)$ & & $\left(M_{\oplus}\right)$ & $\left(M_{\oplus}\right)$ & $\begin{array}{c}\text { min max } \\
\left(\mathrm{cm}^{-3}\right)\end{array}$ & $\begin{array}{c}\text { min }\left(\mathrm{cm}^{-3}\right) \\
\left(\mathrm{cm}^{-2}\right)\end{array}$ & $\left(\mathrm{cm}^{-2}\right)$ \\
\hline solar $^{b}$ & $<5.0 \times 10^{-3}$ & $>3.4$ & $<1.2 \times 10^{-5}$ & $<3.2 \times 10^{-5}$ & 2 & 10 & $5 \times 10^{3}$ & $5 \times 10^{4}$ & $8 \times 10^{14}$ & $2 \times 10^{15}$ \\
$\beta$ Pic $^{c}$ & $<5.7 \times 10^{-5}$ & $>298$ & $<1.6 \times 10^{-5}$ & $<4.1 \times 10^{-5}$ & 6 & 12 & $8 \times 10^{-2}$ & $2 \times 10^{-1}$ & $1 \times 10^{15}$ & $3 \times 10^{15}$ \\
\hline
\end{tabular}

Notes. The gas masses are calculated from $99 \%$ confidence level upper limits on the C II and O I flux. We also list the range of collision partner densities and maximum column densities occurring in the models. ${ }^{(a)}$ Dust-to-gas ratio, using the mm dust mass $M_{\mathrm{mm}}=0.017 M_{\oplus}$ derived by B12. ${ }^{(b)}$ Assuming solar elemental abundances. ${ }^{(c)}$ Assuming elemental abundances similar to the $\beta$ Pic gas disc. ${ }^{(d)}$ Assuming a typical line width of $\sim 2 \mathrm{~km} \mathrm{~s}^{-1}$, the corresponding optical depths are below $10^{-2}$. 